\title{
Friedrich Nietzsche - ein Ahnherr der Psychoanalyse?
}

\author{
Von Gaetano Benedetti
}

\section{Übersicht}

Inhalt dieser Arbeit ist der Versuch, an Hand der Schriften von Friedrich Nietzsche wesentliche Berührungspunkte dieses Autors mit der Psychoanalyse Freuds aufzuzeigen und die These zu vertreten, daß der Philosoph Nietzsche wie kein anderer den Boden vorbereitet hat, auf dem die Psychoanalyse entstanden ist.

Diese These wird aber in der vorliegenden Arbeit auf dem Hintergrund ihrer dialektischen Umkehrung vertreten: Diejenige vom grundsätzlichen Unterschied zwischen dem entlarvenden Denken Nietzsches und der Sorge der Psychoanalyse um den psychisch leidenden Menschen.

\section{Einführung}

Es ist längst bekannt, daß man bei der Lektüre der Schriften Freuds auf Zeilen stößt, die an Nietzsche erinnern, und es umgekehrt bei Nietzsche Stellen gibt, welche dem Psychoanalytiker besonders auffallen.

Die Beziehung Nietzsches zur Psychoanalyse ist ein Thema, über das schon viel geschrieben wurde. Sie ist selbst dem Begründer der Psychoanalyse, Sigmund Freud, nicht entgangen: Von der Nähe der Philosophie Nietzsches berührt oder betroffen, wagte er jedoch weder die Auseinandersetzung mit ihr als einer Grundlage eigener Auffassung noch den Versuch, die Persönlichkeit Nietzsches zu deuten. - Wir wissen nicht, ob das - verdrängte - Bedürfnis nach Priorität ihn an dieser Auseinandersetzung hinderte; vielleicht sträubte sich seine Geistesgröße gegen die (damals naheliegende) Versuchung, die Philosophie von Nietzsche auf psychoanalytische Voraussetzungen zu reduzieren.

In der Schrift «Zur Geschichte der psychoanalytischen Bewegung» meinte Freud (1924), daß er sich «den hohen Genuß der Werke Nietzsches mit der bewußten Motivierung versagt habe, da $\beta$ er in der Verarbeitung der psychoanalytischen Eindrücke durch keinerlei Erwartungsvorstellungen behindert sein wolle». Später, in seiner «Selbstdarstellung» (1925), präzi- 
sierte er: «Ich habe Schopenhauer sehr spät im Leben gelesen. Nietzsche, den anderen Philosophen, dessen Ahnungen und Einsichten sich oft in erstaunlicher Weise mit den mühsamen Ergebnissen der Psychoanalyse decken, habe ich gerade darum lange gemieden; an der Priorität lag mir ja weniger als an der Erhaltung meiner Unbefangenheit.» (S.23)

Seither hat man über Nietzsche und die Psychoanalyse viel geschrieben (z. B.: Rank, 1912; Martin, 1921; Romo, 1935; Dohl, Graber, Mohr, 1941; Brandt, 1955; Bilz, 1958; Weissman, 1963; Tramer, 1967; Marlisz, 1968), wohl immer schwankend zwischen dem Versuch, ihn als geistigen Ahnherr der Psychoanalyse auszulegen, und dem umgekehrten Versuch, ihn zu einem Objekt der Psychoanalyse zu machen, seine Philosophie also als Psychopathologie zu deuten.

In einem gewissen Sinne könnte man dazu auch sagen, daß diese beiden entgegengesetzten Gesichtspunkte in der Spannweite der Persönlichkeit Nietzsches selber liegen, der als ein leidender Philosoph Leiden sowohl deutete, wie ihm auch zum Opfer fiel.

Gerade dieser Gedanke soll uns aber davon abhalten, entweder seine Philosphie auf Psychopathologie zu reduzieren (wie z. B. Reyburn-Hindersks, $1947^{1}$, das tut) oder sich von seiner psychologischen Intuition derart beeindrucken zu lassen, daß man deren Grenzen übersieht.

Nietzsche ist einerseits der geistige Ahnherr der Psychoanalyse gewesen; deren wesentliche Erkenntnis hat er aber verpaßt. So wie ich in meiner ersten, in dieser Zeitschrift erschienenen Abhandlung (Benedetti, 1984) seine Psychopathologie als eine Komponente, aber niemals als die Grundlage seiner Philosophie beschrieben habe, so möchte ich hier dieselbe Dialektik entfalten und die Widersprüchlichkeit der "Ahnherrschaft» erhellen.

Bevor ich auf meine These eingehe, sei vorausgesetzt, daß eine Auseinandersetzung mit der Literatur über «Nietzsche und die Psychoanalyse», die ich vorher angegeben habe, in meinem Text nicht vorgesehen ist - nicht nur wegen des dazu erforderlichen Raumes, sondern auch, weil ich, statt andere zusammenzufassen, einen Aspekt der Problematik beleuchten möchte, der, wie mir scheint, bis heute der Forschung entgangen ist: die Tatsache nämlich, daß die negative Einstellung Nietzsches zur seelischen Schwäche des Menschen ihn zu einem Entlarver machte, der nie im wirklichen Dienste des Mitmenschen aufbaute. Er regte zu einer Vivisektion an, wie er sich selber ausdrückte, die schließlich vor sich selber Halt machen mußte, um nicht eigene Schwäche aufzudecken, zu einem Ideal des Übermenschen 
verführte, das Etwas bei sich und den anderen kompensieren und überwinden wollte, was er nicht lieben konnte.

\section{Der moderne Begriff des Unbewußten bei Nietzsche}

Alle Philosophie ist ein Fragen nach dem Menschen.

Vermutlich hatte aber besonders die Philosophie Nietzsches solches Fragen so ganz in den Mittelpunkt ihres Anliegens gestellt; ich meine ein Fragen, das den Menschen als ein sich suchendes, verirrendes, ablenkendes, sich wiederfindendes Wesen unerbittlich untersuchte.

Eine Ethik der unbedingten philosophisch-psychologischen Wahrheitsforschung gewinnt hier Umrisse, die bereits das Auftauchen der individuell psychologischen und der psychoanalytischen Methode vorausnimmt. Denn hier hören wir unmißverständlich auch das Wort vom Unbewußten, nicht mehr im früheren, romantisch idealisierenden Sinne, sondern im unerschütterlichen Aufsuchen der Gründe.

Während langer Zeit hat man bewußtes Denken als das Denken überhaupt betrachtet: Jetzt erst dämmert uns die Wahrheit auf, daß der größte Teil unseres geistigen Wirkens unbewußt, ungefühlt verläuft (FW 333). Ja, «all unser sogenanntes Bewußtsein» sei ein «mehr oder weniger phantastischer Kommentar über einen ungewußten, vielleicht unwißbaren, aber gefühlten Text» (M 119).

Das Bewußtsein wird hier zum ersten Mal als verschleiernde Abwehr des Unbewußten gesehen:

«Hinterfragen. - Bei allem, was ein Mensch sichtbar werden läßt, kann man fragen: Was soll es verbergen? Wovon soll es den Blick ablenken? Welches Vorurteil soll es erregen? Und dann noch: Bis wie weit geht die Feinheit dieser Verstellung? Und worin vergreift er sich dabei?» (M 523)

Wie der Psychoanalytiker, der den Traum als die «Via Regia» zum Unbewußten betrachtet, erkennt Nietzsche eindeutig, daß die Suche nach dem Unbewußten oft erst durch den Traum erhellt wird:

«Aus dem Traum deuten. - Was man mitunter im Wachen nicht genau weiß und fühlt - ob man gegen eine Person ein gutes oder ein schlechtes Gewissen habe - darüber belehrt völlig unzweideutig der Traum.» (MA II, 76)

«Der Traum und die Verantwortlichkeit. - In allem wollt ihr verantwortlich sein! Nur nicht für eure Träume! Welch elende Schwächlichkeit, welcher Mangel an folgerichtigem 
Mute! Nichts ist mehr euer Eigen als eure Träume! Nichts ist mehr euer Werk! Stoff, Form, Dauer, Schauspieler, Zuschauer - in diesen Komödien seid ihr alles ihr selber! Und hier gerade scheut und schämt ihr euch vor euch, und schon Oedipus, der weise Oedipus, wußte sich Trost aus dem Gedanken zu schöpfen, daß wir nichts für das können, was wir träumen! Ich schließe daraus, daß die große Mehrzahl der Menschen sich àbscheulicher Träume bewußt sein muß. Wäre es anders, wie sehr würde man seine nächtliche Dichterei für den Hochmut des Menschen ausgebeutet haben! - Muß ich hinzufügen, daß der weise Oedipus recht hatte, daß wir wirklich nicht für unsere Träume, aber ebensowenig für unser Wachen verantwortlich sind, und daß die Lehre von der Freiheit des Willens im Stolz und Machtgefühl des Menschen ihren Vater und Mutter hat? Ich sage dies vielleich zu oft: aber wenigstens wird es dadurch noch nicht zum Irrtum.» (M 128)

Nietzsche hatte aber in seinem einsamen Suchen keinen Zugang zum Traum und Unbewußten anderer Menschen.

Es ist zugleich erstaunlich und ergreifend, wie er, der aristokratische Geist auf dem Wege zum Übermenschen, den durchschnittlichen Menschen in sich ansprach und sich hier anschickte, alles in Frage zu stellen, was diesen hätte verstellen können:

«Das Studium des durchschnittlichen Menschen macht ein notwendiges Stück der Lebensgeschichte jedes Philosophen aus, vielleicht das unangenehmste, übelriechendste, an Enttäuschungen reichste Stück.» (J 26)

Nietzsche kleidet seine Intention in ein mythologisches Bild, das uns direkt an das psychoanalytische «Fegen» erinnert: das des Helden Herakles, der den Augiasstall ausmistet:

«Auch heldenhaft. - Dinge vom übelsten Geruch tun, von denen man kaum zu reden wagt, die aber nützlich und nötig sind, - ist auch heldenhaft. Die Griechen haben sich nicht geschämt, unter die großen Arbeiten des Herakles auch die Ausmistung eines Stalles zu setzen.» (M 430)

Wie schwer das ist, auf sich selbst allein angewiesen zu sein, wie diese Aufgabe der Selbsterkenntnis den Menschen ganz in Anspruch nimmt und fast unerfüllbar ist, scheint Nietzsche zu wissen:

«Es gibt Fälle, wo wir die Pferde sind, wir Psychologen und in Unruhe geraten, wir sehen unsern eigenen Schatten vor uns auf- und niederschwanken. Der Psychologe muß von sich absehen, um überhaupt zu sehen.» (GD 947)

Es ist einleuchtend, wie die Auseinandersetzung mit dem Unbewußten diametral entgegengesetzt verlaufen muß zu jener Versenkung des romantischen Geistes in eine das bewußte Selbst mit dem Universum verbindende 
Tiefe der Natur, die man eben unbewußt nannte. - Die Auseinandersetzung bekommt hier einen Sinn, der zum ersten Mal in der Geistesgeschichte als modern angesprochen werden darf: Die ambivalente Beziehung zum eigenen Triebleben.

Die Frage, die ich meine, stellt sich in der Psychoanalyse mit aller Schärfe: Geht es beim Aufdecken darum, die Mächte der Tiefe, die libidinösen Impulse, die erkannten aggressiven Spannungen wahrzunehmen, um sie in einem größeren Wirkungsraum zu integrieren oder um sie besser zu überwinden als in der abspaltenden Verdrängung?

Die Ambivalenz Nietzsches zeigt sich am deutlichsten darin, daß er sich einerseits von dem «Niedrigen, Schmutzigen in der Regel Mensch» abgestoBen fühlt, ja sich über die empört, die nur das Niedrige im Menschen sehen und mit Genuß «im Schmutz wühlen» - wie sich das z. B. im folgenden Satz zeigt:

«Wer das Hohe eines Menschen nicht sehen will, blickt umso schärfer nach dem, was niedrig und Vordergrund an ihm ist - und verrät sich selbst damit» (J 275);

anderseits sich «von einem noch stärkeren Instinkt» von diesem «Niedrigen, Schmutzigen» angezogen fühlt, ja dessen Erkennung als die Wahrheitsleistung erachtet.

Im Gegensatz zum späteren Psychoanalytiker gelingt es Nietzsche aber nicht, die therapeutische, mitmenschliche Nähe zu allem nur allzu Menschlichen zu finden; hier liegt die große Schwäche seiner Erkenntnis.

So bleibt ihm oft nichts anderes übrig, als geradezu sein eigenes Aufdekken als ein "Schnüffeln» auf den Gegner zu projizieren - etwa auf den Priester das verneinte Spiegelbild seines Selbst:

«Diese unanständigste Art der Gegnerschaft, die von hinten und unten, die Hunde-Art, ist insgleichen niemals ausgestorben; die Priester, sofern sie Psychologen sind, haben nie etwas interessanter gefunden, als an den Heimlichkeiten ihrer Gegner zu schnüffeln.» (WzM 396)

Wie unterscheidet sich dieses «Schnüffeln» von der «Vivisektion», mit welcher der Philosoph sein Werk oft vergleicht? Den Sadismus, der im Entschleiern liegt, kann er nicht mit Liebe vermischen; im Zerstören aller tradierten Bilder versteht er sich als Flamme, die alles in Kohle verwandelt; oder er spürt sich als den Zyniker, den er auf seinen Gegner projiziert.

Im Letzten kann die Demaskierung nicht gelingen, wenn die Person hinter der Maske nicht umarmt wird. 
Das Wort «Umarmen» führt uns zu der Frage der Liebe.

Im Unbewußten sieht Nietzsche weniger das Walten jener «Libido», mit deren Erkenntnis die Psychoanalyse ihre ersten Schritte unternahm, sondern vielmehr jene zerstörerische «Grausamkeit», auf deren Verdrängung Kultur und Moral aufbauen; also jener aggressiven Triebfeder, die Freud später unter dem Begriff des Todestriebes subsumierte.

Ich bin auf diesen «Grausamkeitstrieb» bereits in meinem ersten Artikel eingegangen (Benedetti, 1984) und werde mich hier nicht wiederholen. Es sei aber die abschließende Bemerkung gestattet, daß es kein Zufall ist, wenn nicht Libido, sondern Aggressivität ein Grundelement der tiefenpsychologischen Anschauung Nietzsches ist.

Man sieht bei den anderen, was man bei sich selber spürt; und alles Erkennen ist teilweise auch ein Sich-in-die-Welt-Begeben, ein Projizieren. Gerade durch das Fehlen des in der Psychoanalyse so zentralen Libidobegriffes deutet sich jene Lust am Destruktiven an, die einen Autor wie etwa Jaspers, der vom Wirken dieses Philosophen tief beeindruckt war, sagen läßt:

«Die Hauptschwierigkeit ist, daß sein philosophisches Werk von vornherein ein Trümmerhaufen ist.»

\section{Die Lehre von der Abwehr ${ }^{2}$}

Was Freud «Widerstand» genannt hat - also die psychische Kraft, die der Mensch gegen die Selbstwahrnehmung peinlicher Erlebnisinhalte aufrichtet, welche doch zu ihm gehören und die innere Situation des Konfliktes, der eigenen Widersprüchlichkeit heraufbeschwören -, das hat Nietzsche mit einer Klarheit gesehen und mit einer Deutlichkeit ausgedrückt, die nichts zu wünschen übrig läßt. Freilich fehlte Nietzsche noch der systematische Gedankenaufbau, die totalisierende Lehre des psychischen Apparates; dafür gewinnt aber seine Darstellung an Lebensnähe und an unmittelbarer, poetischer Ausdruckskraft:

\footnotetext{
«Der Mensch ist gegen sich selbst, gegen Auskundschaftung und Belagerung durch sich selber sehr gut verteidigt, er vermag gewöhnlich nicht mehr von sich als seine Außenwerke wahrzunehmen. Die eigentliche Festung ist ihm unzugänglich, selbst unsichtbar, es sei denn, daß Freunde und Feinde die Verräter machen und ihn selber auf geheimem Wege hineinführen.» (MA I, 491)

«Sich zu überraschen wissen. - Wer sich selber sehen will, so wie er ist, muß es verstehen, sich selber zu überraschen, mit der Fackel in der Hand. Denn es steht mit dem Geistigen so,
} 
wie es mit dem Körperlichen steht: wer gewohnt ist, sich im Spiegel zu schauen, vergißt immer seine Häßlichkeit: erst durch den Maler bekommt er den Eindruck derselben wieder. Aber er gewöhnt sich auch an das Gemälde und vergißt seine Häßlichkeit zum zweiten Male. - Dies nach dem allgemeinen Gesetze, daß der Mensch das unveränderlich Häßliche nicht erträgt: es sei denn auf einen Augenblick; er vergißt es oder leugnet es in allen Fällen. - Die Moralisten müssen auf jenen Augenblick rechnen, um ihre Wahrheiten vorbringen zu dürfen.» (WuS 316)

«Wie die Knochen, Fleischstücke, Eingeweide und Blutgefäße mit einer Haut umschlossen sind, die den Anblick des Menschen erträglich macht, so werden die Regungen und Leidenschaften der Seele durch die Eitelkeit umhüllt: sie ist die Haut der Seele.» (MA I, 82)

Die Offenheit, mit der reifes menschliches Bewußtsein dem Unbewußten gegenübersteht und Einblick in dieses gewinnt, ruft bei den vielen, die sich davor fürchten und eine unerbittliche Selbst-in-Frage-Stellung meiden, Ablehnung, ja scheinmoralische Entrüstung hervor. Besonders die frühe Psychoanalyse hatte damit zu kämpfen; Freud selber wußte, wie viel Mut und Tapferkeit nötig waren, um nicht in eine innere Verbannung zu gehen. Diese Gefahr kennt auch Nietzsche:

«Wohin die Ehrlichkeit führen kann. - Jemand hatte die üble Angewohnheit, sich über die Motive, aus denen er handelte und die so gut und so schlecht waren wie die Motive aller Menschen, gelegentlich ganz ehrlich auszusprechen. Er erregte erst Anstoß, dann Verdacht, wurde allmählich geradezu verfemt und in die Acht der Gesellschaft erklärt, bis endlich die Justiz sich eines so verworfenen Wesens erinnerte, bei Gelegenheiten, wo sie sonst kein Auge hatte, oder dasselbe zudrückte. Der Mangel an Schweigsamkeit über das allgemeine Geheimnis und der unverantwortliche Hang zu sehen, was keiner sehen will sich selber-, brachten ihn zu Gefängnis und frühzeitigem Tod.» (MA I, 65)

Nicht nur die Psychoanalyse im engeren Sinne, sondern die ganze Tiefenpsychologie gründet in der Auseinandersetzung mit den «dunklen» Aspekten des Unbewußten, in der Aufgabe, sie zu erhellen, sie zu assimilieren, dem Anblick des «Schattens», wie sich Jung ausdrückt, standzuhalten. Wenn einmal manches metapsychologische Gerüst der Psychoanalyse durch die Zeit überholt sein wird, bleibt doch die Lehre von den menschlichen Abwehrmechanismen stehen.

Auf diesem Gebiet kommen sich Nietzsche und Freud nahe; der Unterschied dürfte freilich darin liegen, daß Freud hier die wesentlichen Begriffe wie «Verdrängung», «Projektion» usw. geprägt und somit einen wissenschaftlichen Weg erschlossen hat, wo hingegen Nietzsche ähnliche Phänomene rein deskriptiv, dafür aber mit ungeheurer, fast poetischer Prägnanz schilderte. Wir wollen dies im einzelnen betrachten: 


\section{a) Die Verdrängung}

Die Verdrängung ist eine «Abspaltung vom Ich durch Bewußtseinsentzug», wie Anna Freud im Anschluß an ihren Vater formulierte (A. Freud, 1936). Dieser Entzug erfolgt auf der Grundlage der Selbstwahrnehmung von negativen Emotionen, die von den peinlichen psychischen Inhalten herrühren.

Der Begriff der Verdrängung ist so zentral in der Psychoanalyse, daß keine dynamische Psychologie an ihr vorbeigehen konnte, auch wenn man das Phänomen gelegentlich mit anderen Worten schilderte; Sullivan (1953) sprach z. B. von «selektiver Unaufmerksamkeit», Bilz (1958) von «selektivem Vergessen», usw. Nietzsche formuliert nicht, sondern führt uns direkt auf die Bühne der Handlung:

«Das habe ich getan», sagt mein Gedächtnis. «Das kann ich nicht getan haben» - sagt mein Stolz und bleibt unerbittlich. Endlich - gibt das Gedächtnis nach.» (J 68)

«Man vergißt manches aus seiner Vergangenheit und schlägt es sich absichtlich aus dem Sinn: das heißt, man will, daß unser Bild, welches von der Vergangenheit her uns anstrahlt, uns belüge, unserm Dünkel schmeichle, - wir arbeiten fortwährend an diesem Selbstbetruge.» (MA II, 37)

«Mancher versteht sich darauf, das eigene Gedächtnis zu trüben und zu mißhandeln, um wenigsten an diesem einzigen Mitwisser seine Rache zu haben.» (J 40)

«Sich selbst nicht zu erkennen: Klugheit des Idealisten. Der Idealist: ein Wesen, welches Gründe hat, über sich dunkel zu bleiben, und das klug genug ist, sich auch über diese Gründe noch dunkel zu bleiben.» (WzM 344)

«Der Phantast verleugnet die Wahrheit vor sich, der Lügner nur vor anderen.» (MA II, 6)

Das Verdrängte kann nicht bejaht werden, kann andererseits auch nicht eindeutig aufgegeben werden. Das Verdrängte wird nicht «aus ganzem Herzen», sondern nur halb abgelehnt und noch halb bejaht. Es bleibt bei der für die Neurose charakteristischen ambivalenten Einstellung. Durch die Verdrängung soll Unlust vermieden werden, die eine bewußte Entscheidung unter Umständen mit sich bringen würde. Sie dient der Vermeidung, nicht der Lösung eines Konfliktes. Das Verdrängte wirkt im Unbewußten weiter, weil ihm nicht durch eine bewußte Entscheidung «die Energiebesetzung entzogen» wurde. (Freud, Selbstdarstellung XIV, 55)

Nietzsche hat sich mehrmals dazu geäußert, daß verdrängte Affekte stärker werden und eine unheilvollere Wirkung haben als frei geäußerte («abreagierte»): 
«Sich rächen wollen und sich rächen. - Einen Rachegedanken haben und ausführen heißt einen heftigen Fieberanfall bekommen, der aber vorübergeht; einen Rachegedanken aber haben, ohne Kraft und Mut ihn auszuführen, heißt ein chronisches Leiden, eine Vergiftung an Leib und Seele mit sich herumtragen. Die Moral, welche nur auf die Absichten sieht, taxiert beide Fälle gleich: für gewöhnlich taxiert man den ersten Fall als den schlimmeren (wegen der bösen Folgen, welche die Tat der Rache vielleicht nach sich zieht). Beide Schätzungen sind kurzsichtig.» (MA I, 60)

«Neid mit oder ohne Mundstück. - Der gewöhnliche Neid pflegt zu gackern, sobald das beneidete Huhn ein Ei gelegt hat, er erleichtert sich dabei und wird milder. Es gibt aber einen noch tieferen Neid: der wird in solchem Fall totenstill, und, wünschend, daß jetzt jeder Mund versiegelt würde, immer wütender darüber, daß dies gerade nicht geschieht. Der schweigende Neid wächst im Schweigen.» (MA II, 53)

«Der Zorn als Spion. - Der Zorn schöpft die Seele aus und bringt selbst den Bodensatz ans Licht. Man muß deshalb, wenn man sonst sich nicht Klarheit zu schaffen weiß, seine Umgebung, seine Anhänger und Gegner in Zorn zu versetzen wissen, um zu erfahren, was im Grunde alles wider uns geschieht und gedacht wird.» (MA II, 54)

\section{b) Die Rationalisierung}

Man kann der Konfrontation mit den wahren Gründen des eigenen Verhaltens auch dadurch ausweichen, daß man diesem eine schein-rationale Begründung unterschiebt. Eine solche scheinrationale Begründung eines nicht-rationalen Verhaltens nennt die Psychoanalyse eine «Rationalisierung». Charakteristisch für eine solche Scheinbegründung ist, daß sie erst nachträglich dem Verhalten unterschoben wird. Außerdem verläuft sie unbewußt. Man betrügt damit also nicht nur andere, sondern vor allem auch sich selbst.

So sagt Nietzsche von den Philosophen:

«Sie stellen sich sämtlich, als ob sie ihre eigentlichen Meinungen durch die Selbstentwicklung einer kalten, reinen, göttlich unbekümmerten Dialektik entdeckt -, erreicht hätten, ... während im Grunde ein vorweg genommener Satz, ein Einfall, eine 〈Eingebung〉, zumeist ein abstrakt gemachter und durchgesiebter Herzenswunsch von ihnen mit hinterher gesuchten Gründen verteidigt wird.» (J 5)

Nietzsche sieht dieses Phänomen in dem größeren Rahmen des Verhältnisses von Instinkt und Vernunft. Der Instinkt handelt, ohne von sich selbst zu «wissen» und ohne nach «Gründen» zu fragen. Die Vernunft kommt hinterher mit nachträglichen logischen Begründungen und Rechtfertigungen. 
«Ihr Instinkt ist unwiderlegt, - was gehen uns ihre rückläufigen Schleichwege an!» (J 10)

«Man muß den Instinkten folgen, aber die Vernunft überreden, ihnen dabei mit guten Gründen nachzuhelfen.» (J 191)

Das sei die eigentliche Falschheit und Selbstüberlistung des Sokrates gewesen. «Die Vernunft ist nur ein Werkzeug.» (ebd.)

«Die Moral sagt: ich brauche manche Antworten, Gründe, Argumente; - Skrupel mögen hintendrein kommen oder auch nicht.» (WzM 423, vgl. auch WzM $430 \mathrm{ff}$.)

Nietzsche gibt dazu eine Fülle anschaulicher Beispiele aus dem täglichen Leben. - Da ist zunächst die Reaktion des Fuchses, dem die Trauben, die ihm zu hoch hängen, zu sauer sind. Nietzsche erweitert sie noch um eine Variante:

«Ein rechter Fuchs nennt nicht nur die Trauben sauer, welche er nicht erreichen kann, sondern auch die, welche er erreicht und andern vorweggenommen hat.» (WuS 244)

Er nennt die Saure-Trauben-Reaktion an anderer Stelle den «Kunstgriff des Entsagenden»:

«Wer gegen die Ehe protestiert ... wird diese nach ihrer niedrigsten gemeinsten Auffassung zu verstehen suchen. Ebenso wer die Ehre bei den Zeitgenossen von sich abweist, wird deren Begriff niedrig fassen; so erleichtert er sich die Entbehrung und den Kampf dagegen ...» (MA I, 598)

Man kann die eigene Unfähigkeit, ein gewünschtes Ziel zu erreichen, auch umgekehrt dadurch motivieren und verdecken, daß man das (heimlich) Erstrebte übermäßig in «unerreichbare» Höhen emporhebt und dabei den Bescheidenen spielt, während unter der Oberfläche der Neid glimmt.

«Kultus des Genius aus Eitelkeit. - Weil wir gut von uns denken, aber doch durchaus nicht von uns erwarten, daß wir jeden Entwurf eines Raffaelischen Gemäldes oder eine solche Szene wie die eines Shakespeareschen Dramas machen könnten, reden wir uns ein, das Vermögen dazu sei ganz übermäßig wunderbar, ein ganz seltener Zufall, oder, wenn wir noch religiös empfinden, eine Begnadigung von oben. So fördert unsere Eitelkeit unsere Selbstliebe den Kultus des Genius: denn nur wenn dieser ganz fern von uns gedacht ist, als ein miraculum verletzt er nicht (selbst Goethe, der Neidlose, nannte Shakespeare seinen Stern der fernsten Höhe; wobei man sich jenes Verses erinnern mag: die Sterne, die begehrt man nicht).» (MA I, 162)

«Seine Armut motivieren. - Wir können freilich durch kein Kunststück aus einer armen Tugend eine reiche, reich fließende machen, aber wohl können wir ihre Armut schön in die Notwendigkeit umdeuten, so daß ihr Anblick uns nicht mehr wehtut und wir ihrethalben dem Fatum keine vorwurfsvollen Gesichter machen. So tut der weise Gärtner, der das 
arme Wässerchen seines Gartens einer Quellnymphe in den Arm legt und also die Armut motiviert - und wer hätte nicht gleich ihm die Nymphen nötig!» (FW 17)

«Anmaßung als letztes Trostmittel. - Wenn man ein Mißgeschick, seinen intellektuellen Mangel, seine Krankheit sich so zurechtlegt, daß man hierin sein vorgezeichnetes Schicksal, seine Prüfung oder die geheimnisvolle Strafe für früher Begangenes sieht, so macht man sich sein eigenes Wesen dadurch interessant und erhebt sich in der Vorstellung über seine Mitmenschen. Der stolze Sünder ist eine bekannte Figur in allen kirchlichen Sekten.» (MA I, 590)

Das Gegenstück zur «Saure-Trauben-Reaktion» ist die «Süße-ZitronenReaktion», die sich über Mißgeschicke dadurch zu trösten versucht, daß sie «das Übel in ein Gut umdeutet». (MA I, 108)

«Wir verstehen uns ganz gut darauf, Süßigkeiten auf unsere Bitternisse zu träufeln.» (FW 326)

«Trostgründe. - Bei einem Todesfall braucht man zumeist Trostgründe, nicht sowohl um die Gewalt des Schmerzes zu lindern, als um zu entschuldigen, daß man sich so leicht getröstet fühlt.» (MA I, 510)

Antipathie und $\mathrm{Haß}$ gegen andere versucht der Mensch mit Gründen zu motivieren, die mehr im Objekt als im Subjekt des Hasses liegen. Das geht bereits über in das, was wir im nächsten Abschnitt als «Projektion» besprechen werden.

«Gründe und ihre Grundlosigkeit. - Du hast eine Abneigung gegen ihn und bringst auch reiche Gründe für diese Abneigung vor - ich glaube aber nur deiner Abneigung und nicht deinen Gründen! Es ist eine Schöntuerei vor dir selber, das, was instinktiv geschieht, dir und mir wie einen Vernunftsschluß vorzuführen.» (M 358)

«Es mißfällt mir.» - Warum? - «Ich bin ihm nicht gewachsen.» - «Hat je ein Mensch so geantwortet?» (J 185) (Vgl. auch MA II, 391, wo derselbe Gedanke in noch nicht so ausgereifter Formulierung auftaucht.)

Etwas geradezu Alltägliches ist es, ein eigenes Versagen oder Mißgeschick mit einem Schuldigen, den man sich in der Umwelt sucht, zu motivieren.

«Mit Vorteil angefeindet. - Menschen, welche der Welt ihre Verdienste nicht völlig deutlich machen können, suchen sich eine starke Feindschaft zu erwecken. Sie haben den Trost zu denken, daß diese zwischen ihren Verdiensten und deren Anerkennung stehe und daß mancher andere dasselbe vermute, was sehr vorteilhaft für ihre Geltung ist.» (MA I, 567)

«Wer eine Partei innerlich stärken will, biete ihr Gelegenheit, um ersichtlich ungerecht behandelt werden zu müssen; dadurch sammelt sie ein Kapital guten Gewissens, das ihr vielleicht bis dahin fehlte.» (MA II, 306) 
Freilich sind bei Freud und Nietzsche die «Nuancen» verschieden. Freud betont die Verdrängung von Triebansprüchen, worunter er, hier ganz im Unterschied zu Nietzsche, primär sie libidinös versteht. Der Philosoph drängt nicht zu den triebpsychologischen Mechanismen, er bleibt vielmehr beim Phänomen des durch die peinlichen Inhalte verletzten Stolzes - was dem heute geläufigen Begriff der narzißtischen Kränkung nahe kommt.

Schließlich können nicht nur Erkenntnisse über sich selbst, sondern auch Erkenntnisse über die Realität schlechthin, falls diese für das Ich unerträglich wird, abgewehrt werden. Ein solches «Nicht-wahrhaben-Wollen» der Realität heißt in der psychoanalytischen Terminologie "Verleugnung». ${ }^{3}$

$\mathrm{Da}$ es auch in der Politik sehr oft «erwünschte Feinde» gibt, die einen willkommenen Vorwand für eigene Gewalttaten liefern, ist altbekannt.

«Zu allem, was solche Regierungen öffentlich hassen, haben sie jetzt eine heimliche Zuneigung und Innigkeit: sie müssen ihre Seele verschleiern.» (MA II, 316)

«Das Leben des Feindes - wer davon lebt, einen Feind zu bekämpfen, hat ein Interesse daran, daß er am Leben bleibt.» (MA I, 531)

«Bedingung des Heroentums. - Wenn einer zum Helden werden will, so muß die Schlange vorher zum Drachen geworden sein, sonst fehlt ihm sein rechter Feind.» (MA I, 498)

«Schwelgerei der Rache. - Grobe Menschen, welche sich beleidigt fühlen, pflegen den Grad der Beleidigung so hoch als möglich zu nehmen und erzählen die Ursache mit stark übertreibenden Worten, um nur in dem einmal erweckten Haß- und Rachegefühl sich recht ausschwelgen zu können.» (MA I, 62)

\section{c) Die Projektion}

Damit sind wir bereits zu einem weiteren Abwehrmechanismus übergegangen, nämlich der Projektion: Unbewußte Inhalte, die dem Ich unannehmbar sind, können nach außen verlegt werden. Sie scheinen dann in der Außenwelt ein selbständiges Dasein zu führen, da der Zusammenhang mit dem Subjekt, dem sie entstammen, nicht mehr wahrgenommen wird.

Die einfachste Form der Projektion ist die Nach-außen-Verlegung von Stimmungen und Affekten. Wir sehen dann die Außenwelt getrübt durch die Brille der eigenen Zustände.

«Liebe und Haß sind nicht blind, aber geblendet vom Feuer, das sie selber mit sich tragen.» (MA I, 566) 
Nach außen verlegte Wunschbilder führen zur Idealisierung der Wirklichkeit mit all ihren Illusionen und Sentimentalitäten. (Vgl. MA I, 279; MA II, 345 ; WuS, 265.)

Unsere mitmenschlichen Beziehungen sind nur allzu oft von solchen Projektionen überlagert. Wir sehen dann im andern Dinge, die gar nicht zu ihm gehören, sondern aus uns selbst stammen.

«Jünglinge wechseln in bezug auf dieselbe Person mit Hingebung und Unverschämtheit ab: weil sie im Grunde nur sich in dem andern verehren und verachten, und zwischen beiden Empfindungen in bezug auf sich selber hin- und hertaumeln müssen. ...» (MA II, 277)

Handelt es sich um Menschen, in die wir unsere unerfüllten Wünsche verlegen, dann werden diese «vergöttert». Dem Betreffenden (oder Betroffenen) wird dabei ein Bild aufgedrängt, dem er nicht entsprechen kann und gegen das er schließlich protestiert. (M 279; M 298; M 379)

Eigene Wünsche, die man sich selbst nicht eingesteht, können auf andere Menschen projiziert werden, wobei man sich gleichzeitig mit ihnen identifiziert. Man behandelt andere dann so, wie man eigentlich selbst gerne behandelt werden möchte. Der eigene $\mathrm{Wunsch}$ wird am andern befriedigt, so daß der Vorwurf des Egoismus nicht gefürchtet werden muß. A. Freud nennt diesen Vorgang die «altruistische Abwehr von Triebansprüchen». (Das Ich und die Abwehrmechanismen)

Hierzu Nietzsche:

«Wir lieben im andern unsere Hoffnungen.»

«Unser Glaube an andere verrät, worin wir gerne an uns selbst glauben würden.» (Z)

Außer unseren unerfüllten $W$ ünschen ist es vor allem unsere Schuld, die wir nach außen projizieren. Der Betroffene wird dadurch nicht vergöttert, sondern eher verteufelt. Wir richten nicht unsere Wünsche, sondern unseren $\mathrm{Ha}$ a au ihn - einen $\mathrm{Ha}$, der eigentlich uns selbst gelten müßte.

«Unmut über andere und die Welt. - Wenn wir, wie so häufig, unsern Unmut an anderen auslassen, während wir ihn eigentlich über uns empfinden, erstreben wir im Grunde eine Umnebelung und Täuschung unseres Urteils: wir wollen diesen Unmut a posteriori motivieren, durch die Versehen, Mängel der andern, und uns selber so aus den Augen verlieren. - Die religiös strengen Menschen, welche gegen sich selbst unerbittliche Richter sind, haben zugleich am meisten Übles der Menschheit überhaupt nachgesagt: ein Heiliger, welcher sich die Sünden und den andern die Tugenden vorbehält, hat nie gelebt; ebenso wenig wie jener, welcher nach Buddhas Vorschriften sein Gutes vor den Leuten verbirgt und sie sein Böses allein sehen läßt.» (MA I, 607) 
Wie glänzend ist die Definition:

«Verleumdungen sind Krankheiten anderer, die an deinem Leibe ausbrechen, so daß du an dir die Kur vornehmen kannst, die den anderen nützen soll.» (WuS, 264, vgl. auch MA I, 562 und M 413: Der Ankläger enthüllt seinen eigenen Charakter)

Besonders im Krieg feiern solche Projektionen Triumphe. Was wir Haß und Feindschaft nennen, beruht zum großen Teil darauf, daß die Schattenseiten der eigenen Partei auf den sogenannten «Feind» projiziert werden. So entsteht die typische «Schwarz-weiß-Malerei» oder Karikierung des Gegners, durch die dieser verächtlich gemacht wird bei gleichzeitiger eigener Selbstüberschätzung.

Das Gegenstück zur Projektion ist die Introjektion. In der Projektion sucht der Betreffende die Schuld beim andern. In der Introjektion sucht er sie nur bei sich selbst und nimmt auch die Schuld anderer auf sich, weil er nicht wagt, sie dem andern zu präsentieren. Alle Kräfte, mit denen man sich gegen andere durchsetzt und sich gegen fremde Angriffe und Beschuldigungen wehrt (nach Freud die Aggression), werden als schuldhaft empfunden und sind gehemmt. Aus diesem Schuldgefühl entsteht Angst. Die Folge einer solchen Introjektion einer äußeren Kritik kann dann wieder eine Projektion sein. Die durch eine solche verinnerlichte Kritik unterdrückten Impulse werden nach außen projiziert, beispielsweise wenn jemand, der sich selbst schlecht behandelt oder hart kritisiert, sich von andern mißhandelt, kritisiert oder verfolgt fühlt.

Ein Beispiel für eine Introjektion ist etwa die übertriebene Bewunderung anderer aus Selbstunsicherheit. (MA II, 355)

\section{d) Überkompensation}

Man kann seine Schwächen durch übertriebene Betonung des Gegenteils vor andern und vor sich selbst zu verbergen suchen.

«Leidenschaft hat Recht. - Niemand spricht leidenschaftlicher von seinem Rechte als der, welcher im Grunde seiner Seele einen Zweifel an seinem Rechte hat. Indem er die Leidenschaft auf seine Seite zieht, will er den Verstand und dessen Zweifel betäuben: so gewinnt er das gute Gewissen und hat mit ihm den Erfolg bei den Mitmenschen.» (MA I, 597)

«Die freiwilligen Blinden. - Es gibt eine Art schwärmerischer, bis zum Äußersten gehender Hingebung an eine Person oder Partei, die verrät, daß wir im Geheimen uns ihr überlegen 
fühlen und darüber mit uns grollen. Wir blenden uns gleichsam freiwillig zur Strafe dafür, daß unser Auge zuviel gesehen hat.» (M 414)

Die Überkompensation entsteht hier auf dem Boden einer Verdrängung. Wir wollen die Schwächen nicht wahrhaben und kehren deshalb um so mehr die positiven Seiten heraus. Die Übertreibung kommt in der affektiven Beteiligung zum Ausdruck («schwärmerische, bis zum Äußersten gehende Hingebung»).

Eine solche übertriebene Hingabe kann eine Überkompensation gerade gegenteiliger, aber verdrängter Tendenzen sein. Man denke etwa an den Typ der «überprotektiven Mutter», die durch ihre Überbesorgtheit sich selbst und andere davon ablenken will, daß ihr das Kind, dem diese Sorge gilt, im Grunde unerwünscht war.

«Die «Gewissenhaften». - Habt ihr acht gegeben, was für Menschen am meisten Wert auf strengste Gewissenhaftigkeit legen? Die, welche sich vieler erbärmlicher Empfindungen bewußt sind, ängstlich von sich und an sich denken und Angst vor anderen haben, die ihr Inneres so sehr wie möglich verbergen wollen, - sie suchen sich selber zu imponieren, durch jene Strenge der Gewissenhaftigkeit und Härte der Pflicht, vermöge des strengen und harten Eindrucks, den andere von ihnen dadurch bekommen müssen (namentlich Untergebene).» (M 233)

Nietzsche schildert hier andeutungsweise den Typ des Angstneurotikers, dem die Gewissenhaftigkeit dazu dient, die zugrunde liegende Unsicherheit und die damit verbundene Angst zu kompensieren. Hinter der Angst können verdrängte Schuldgefühle stecken. Das schlechte Gewissen soll durch betonte Gewissenhaftigkeit ausgeglichen werden.

«Worauf phantastische Ideale raten lassen. - Dort, wo unsere Mängel liegen, ergeht sich unsere Schwärmerei ...» (MA 377)

Nietzsche ist der Überzeugung, daß alle «Ideale» solche Überkompensationen von Schwächen, man kann auch sagen Phantasiebefriedigungen oder Wunschprojektionen sind. Er will sogar den Begriff «Ideal» einfach durch «Wünschbarkeit» ersetzen (WzM 330), bzw. die Ideale als Komplement von Schwächen auffassen.

«Wenn die moralischen Werte die höchsten Werte geworden sind, so verrät dies, daß das moralische Ideal das unerfüllteste gewesen ist.» (WzM 335, 336)

Auch der Traum ist für Nietzsche eine Kompensation unerfüllter Wünsche. 
«Der Hunger gibt sich nicht mit geträumter Speise zufrieden; aber die meisten Triebe, namentlich die sogenannten moralischen, tun gerade dies, - wenn meine Vermutung erlaubt ist, daß unsere Träume eben den Wert und Sinn haben, bis zu einem gewissen Grade jenes zufällige Ausbleiben der (Nahrung) während des Tages zu kompensieren.» (M 119)

«Welche Bestialität der Idee bricht sofort heraus, wenn sie nur ein wenig verhindert wird, Bestie der Tat zu sein.» (GdM II, 22)

\section{Die Grenze in der Kommunikation Nietzsches in der Gegenüberstellung zur Psychoanalyse}

Die fehlende Synthese von entlarvender Psychologie mit psychotherapeutischem Interesse für die menschliche Not, die sich in den psychologischen Widerständen versteckt, ist vielleicht das Merkmal, das Nietzsche am eindrücklichsten vom psychoanalytischen Anliegen trennt. Oft stößt er auf psychodynamische Fragestellungen, allerdings in erster Linie dann, wenn es ihm darum geht, etwas in abwertender Weise für psychopathologisch zu erklären (J 47; WzM 47; WzM 234; GdM III, 17).

Hie und da gelingen ihm tiefe psychotherapeutische Einblicke; im Wort etwa: «Arzt, hilf Dir selber; so hilfst du auch deinem Kranken noch. Da sei seine beste Hilfe, daß er den mit den Augen sehe, der sich selber heil macht» (Z 339), hat er eine wesentliche psychotherapeutische Aufgabe erblickt selber verwirklichen konnte er sie aber nicht.

Man bekommt manches Mal den Eindruck, daß Nietzsche mit einem gewissen Neid auf die Seelsorger und Priester-Ärzte früherer Zeiten herabblickt. Er möchte ihnen etwas Besseres an die Seite stellen, aber er erschöpft sich im Aphorismus: «Wo sind die neuen Ärzte der Seele?» (M 52) in fruchtloser Polemik (siehe auch MA I, 243; GdM $17 \mathrm{ff}$.).

Seine Worte geben oft ein trostloses Bild vom Menschlichen wieder:

«Der Mensch, eingesperrt in einen eisernen Käfig von Irrtümern, eine Karikatur des Menschen geworden, krank, kümmerlich, gegen sich selbst böswillig, voller $\mathrm{Haß}$ auf die Antriebe zum Leben, voller Mißtrauen gegen alles, was schön und glücklich ist am Leben, ein wandelndes Elend: diese künstliche, willkürliche nachträgliche Mißgeburt ...» (WzM 397)

Ist eine solche Erkenntnis durch das Ziel der Überwindung befreiend, oder wendet sich seine Erkenntnis des Erkennens als Waffe gegen sich selbst? 
«... Im Erkennenwollen ist Habsucht und Erobern; im Künstler tritt die zurückgetretene Verstellungs- und Lügenkraft auf; die Triebe werden zu Dämonen umgeschaffen, mit denen es Kampf gibt.» (WzM 376)

Durch sein Anliegen der Erschütterung menschlicher Widerstände, die sich als vermeintliche Werte gebärden, trifft Nietzsche in seinen leidenschaftlichen solipsistischen Auseinandersetzungen nie auf die wirklichen Menschen, die sich dahinter verstecken. Diese wirklichen Menschen, wie sie der wahrhaftige Arzt kennt, bauen einerseits ihre verhängnisvollen Widerstände auf, um dem Elend dieses Lebens auszuweichen; und sie nehmen anderseits unwissend und unwillentlich dadurch ein noch größeres, einzelnes oder kollektives Unglück in Kauf.

Ich meine hier vor allem den leidenden Menschen, dessen von Nietzsche angeprangerte «Grausamkeit» nur Selbstzerstörung ist, und dessen ideologische Selbsttäuschung wesensmäßig nichts anderes als die des Übermenschen ist; den leidenden Menschen, welcher immer das eigentliche Objekt und Subjekt der Psychoanalyse bleibt; denjenigen also, welcher am Ende der InFrage-Stellung, durch den Verlust seiner sich als Widerstände gegen Wandlung und Wahrheitserforschung gebenden Sicherungen zunächst erst recht zum psychotherapeutischen Objekt werden will.

So erinnere ich mich an meine Lektüre von Nietzsche in allerersten jungen Jahren: Faszinierende In-Frage-Stellungen, tiefsinnige Erschütterungen ließen ein Trümmerfeld übrig, keine wirklichen Hoffnungen, es sei denn solche, die in der Verdrängung der Schwächen aus «übermenschlichen Phantomen» wuchsen.

Nietzsche kämpft dauernd mit Widersachern, sieht aber den Mitmenschen nicht, der mit erschütterten Widerständen brach liegt, auf daß er mit Hilfe eines dialogischen Partners aus der «Umwertung aller Werte» die Kraft zum Sprunge zu seinem eigentlichen, authentischen Selbst hole. Das Anliegen der Psychoanalyse mündet dagegen in einer Verbundenheit, die Freud die Neurose als einen würdigen Gegner ansprechen ließ und die uns später sogar von der Kompetenz des Patienten im gemeinsamen Verstehen seines Anliegens sprechen ließ.

Eine solche Verbundenheit des Geistes, die in der Geschichte der Philosophie als konkretes dialogisches Bündnis mit Sokrates und Plato, und in unserem wissenschaftlichen Zeitalter erst durch die Psychoanalyse auftauchte, fehlt meiner Meinung nach in der philosophischen Planung Nietzsches. Denn sein erhellendes Ziel war doch nicht die wohlmeinende Begegnung mit den auch im Widerstand ernst zu nehmenden Seiten des Mitmen- 
schen; sondern im frontalen Angriff im Hinblick auf ein letztes Endes narzißtisches Ideal der Vollkommenheit: der Übermensch.

Man beachte etwa, als ein Beispiel für viele, folgende Stellungnahme:

«Die Schwachen und Mißratenen sollen zugrunde gehen: erster Satz unserer Menschenliebe. Und man soll ihnen noch dazu helfen. Was ist schädlicher als irgendein Laster? Das Mitleiden der Tat mit allen Mißratenen und Schwachen.» (A., Aphor.2)

Eine derartige Paradoxie, daß nämlich ein wichtiger Vorläufer der Psychoanalyse, der Entdecker des Unbewußten im modernen Sinne, der Entdecker der verfälschten psychologischen Abwehr, beim völligen Fehlen der philosophisch-liebenden und ärztlichen Sicht doch eine im Grunde seelentherapeutisch fragwürdige Intention entwickeln mußte, gehört zum tragischen Scheitern der Person Nietzsche; jener großartigen Person, die der existentialistische Philosoph Abbagnano die "personalità impossibile», die «unmögliche Persönlichkeit», genannt hat, welche nämlich im Konflikt zwischen übermenschlichem Ideal und Scheitern im Wahnsinn unterging.

«Il tentativo di divinizzare l'uomo ... ha subito lo scacco definitivo nella personalità stessa di chi lo ha compiuto.» (Abbagnano, 1982)

Nietzsche vermag nicht, seinen Widersacher als tragisch Leidenden zu sehen, weil er seine Patientenseite nicht wahrnimmt und von ihm den Entwurf zum grandiosen Ich, zum Übermenschen, erwartet; er überfordert, wo er auffordert.

In der Gegenüberstellung zu dieser Sicht möchte ich abschließend behaupten, das Wesen der Psychoanalyse sei die Aufdeckung der unbewußten Wurzeln des Leidens, die in den Symptomen einer mißlungenen Sozialisierung stecken; die Psychoanalyse wolle den Menschen erschüttern, ihn bescheiden machen und ihn dadurch zu der von uns, von unsern Plänen und Zielen verfügbaren Größe zurückgeben.

Sie will auch die Abwehr stehen lassen, sofern diese sublimiert und in den Dienst der allgemeinen Lebensinteressen gestellt werden kann. 


\section{Anmerkungen}

${ }^{1}$ «Was Nietzsche der Welt vorhielt, war sein eigenes Leiden. Das Ressentiment, der Sklavenaufstand, die ewige Zerstörung und Verwandlung, die Herrschsucht, der Lebensneid, der Wille zur Macht: das alles war Nietzsche selbst.» (Reyburn-Hindersks, 1947)

${ }^{2}$ In diesem Kapitel habe ich Teile eines älteren (1970), nicht publizierten, mir überlassenen Manuskriptes meines früheren Mitarbeiters W. Barck verwendet. Ich möchte ihm hier meinen besten Dank aussprechen.

${ }^{3}$ «Die Verdrängung richtet sich gegen Wahrnehmungen von innen, die Leugnung gegen solche von außen.» (A. Freud, 1936)

\section{Abkürzungen}

A Der Antichrist (1888).

MA I Menschliches, Allzumenschliches: Ein Buch für freie Geister, Band I (1878).

MA II dasselbe, Band II: Vermischte Meinungen und Sprüche (1879) (ohne «Der Wanderer und sein Schatten»).

WuS Der Wanderer und sein Schatten (1880).

M Morgenröte, Gedanken über moralische Vorurteile (1881).

FW Die fröhliche Wissenschaft (1882).

Z Also sprach Zarathustra: Ein Buch für Alle und Keinen (1883-85).

J Jenseits von Gut und Böse, Vorspiel einer Philosophie der Zukunft (1886).

GdM Zur Genealogie der Moral: Eine Streitschrift (1887).

$\mathrm{W}_{\mathrm{z}} \mathrm{M} \quad$ Der Wille zur Macht, Versuch einer Umwertung aller Werte.

Mus Musarion-Ausgabe.

\section{Literatur}

1 Abbagnano, N.: Storia della filosofia, vol. III, p. 379. Utet, Turin 1982.

2 Benedetti, G.: Die neurotische Lebensproblematik Nietzsches als eine Wirkkraft und eine Grenze seiner Philosophie. Gesnerus, vol.41, H.1/2 (1984) 111-132.

3 Bilz, R.: Der Verdrängungsschutz: Eine Untersuchung über das Paradigma der Verdrängung bei Nietzsche und bei Freud. Nervenarzt 29 (1958) 145-148.

4 Brandt, R. I.: Freud and Nietzsche: a comparison. Rev. Un. Ottawa, 25 (1955) 225-234.

5 Dohl, W., Graber, G.H., Mohr, F.: Leibniz, Carus und Nietzsche als Vorläufer unserer Tiefenpsychologie. Zentralblatt für Psychotherapie und ihre Grenzgebiete einschließlich der medizinischen Psychologie und psychischen Hygiene, No.3, 1941.

6 Freud, A.: The Ego and the Mechanisms of Defence. In: The Writings of Anna Freud, vol.2. International Universities Press, 1936.

7 Freud, S.: Zur Geschichte der psychoanalytischen Bewegung. Gesammelte Schriften, Bd.IV. Internationaler psychologischer Verlag, Leibzig 1924.

8 Freud, S.: Die Traumdeutung. Internationaler psychoanalytischer Verlag, Leipzig 1925. 
9 Freud, S.: Selbstdarstellung. Gesammelte Schriften, Bd.XI. Internationaler psychoanalytischer Verlag, Leipzig 1925.

10 Jaspers, K.: Zu Nietzsches Bedeutung in der Geschichte der Philosophie. In: K. Jaspers, Aneignung und Polemik, S.390. Piper, München 1968.

11 Koureta, D.: Nietzscheisen and Freudinisen. Hellenike Iatrike 29 (1960) 437-456.

12 Marlisz, B.: Freud and Nietzsche. The Psychoanalytic Revue 55 (1968) 360-375.

13 Martin, E.D.: Friedrich Nietzsche: eine psychoanalytische Studie. Internationale Zeitschrift für ärztliche Psychoanalyse 7 (1921) 517-518.

14 Rank, O.: Nietzsche. Zentralblatt für Psychoanalyse und Psychotherapie 2 (1912) 473.

15 Reyburn-Hindersks, F.: Nietzsche. Ein Menschenleben und seine Philosophie. 1947.

16 Romo, F.G.: Nietzsche, précurseur de la psychoanalyse. Evolution psychiatrique 5 (1) (1935) 55-78.

17 Sullivan, H.S.: The Interpersonal Theory of Psychiatry. Norton, New York 1953.

18 Tramer, F.: Friedrich Nietzsche und Sigmund Freud. Jahrbuch für Psychologie und Psychotherapie 7 (1967) 325-350.

19 Weissmann, K.: Nietzsche and the anti-maturism. American Imago 20 (1963) 315-329.

\section{Summary}

This paper presents important points of contact between the writings of Friedrich Nietzsche with the Psychoanalysis of Freud and suggests that the philosopher Nietzsche, as no else, prepared the ground upon which Psychoanalysis arouse. This thesis is presented, however, against the background of its dialectical negation or limitation, because of the basic difference between the deep concern of Psychoanalysis with the psychically suffering human beeing and the "uncovering" thinking of Nietzsche regardless of a true love for what we consider the fragility of human existence.

Prof. Gaetano Benedetti

Inzlingerstraße 271

CH-4125 Riehen 